\title{
Effects of EM Radiation on Living System
}

\author{
P.gauthamipriyadarshini ${ }^{1}$, C.H.R.Phani kumar $^{2}$ \\ ${ }^{I}$ (Second M.tech RF and MICROWAVE ENGINEERING, GITAM university, Visakhapatnam, India) \\ ${ }^{2}$ (Assistant Professor/ Dept of ECE, GITAM University, Visakhapatnam,, India)
}

\begin{abstract}
Electrical excitability and signaling frequently associated with rapid responses to environmental stimuli, as well as in some algae and some higher plants. The presence of electrical signal, such as action potentials (AP) in plant cells suggested that plant cells, too, make use of ion channels to transmit information over long distances. In the light of rapid progress in plant biology during the past decade, the assumption the electrical signals donot only trigger rapid leaf movements in sensitive plant such as mimosa pudica, the electromagnetic radiations may also cause effect on mechanical and electrical stimulis. Summarizing recent progress in the field of electromagnetic radiation on plants, the present review will focus on the mechanical and electrical stimuli of plants.
\end{abstract}

Keywords - Electromagnetic radiation, Electrical signal, Mimosa pudica, Mechanical and electrical stimulis.

\section{INTRODUCTION}

Mobile phone companies often sell more batteries than phones to consumers. The phones sold to user include a rechargeable battery so that the device is immediately useful, but a certain number of consumers are expected to own more than one battery during the life of their phone. The same can probably be said for laptops and camcorders. Yet, there is little incentive for consumers to buy new batteries except for when they fail or when the consumer feels the need for a larger battery. Unlike other areas of mobile computing that benefit from exponential improvements in performance, battery energy density changes slow that there is little pressure for consumers to upgrade. Wireless connectivity is also a conundrum for mobile designers. While the designer can control the CPU, RAM, disk, and battery in his device, wireless connectivity is often provided by another party. In the extreme case, a wireless provider may go out of business and significantly impact the quality of service that can be expected. Even on a minute - by - minute basis a, wireless connection may or may not be available at any given moment. The device designer must either cache information for the user or refuse service when the network is not available. Many RF transmitters of various sorts distributed throughout today's urban environments, one might consider background RF as a potential power reservoir for mobile devices. Electronic systems that harvest energy from ambient radiation sources, however, tend to be extremely power-limited and generally require a large collection area or need to be located very close to the radiated sources. A classic example can be found in old -fashioned crystal radio kits that draw their power directly from Am radio stations, which play audibly through high - impedance headphones without needing a local source of energy. The size of the require antenna, however can be prohibitive for wearable applications unless the bearer is very close to the transmitter, and access to a good ground is usually required. Capturing enough energy from vibrations to power sensors and telemetry has a long history in vehicles, where considerable mechanical excitation is usually available. In the patent literature, one can find techniques ranging from linear motor generators with bouncing spring- mounted magnet arrays for use in trucks and trains to piezoelectric generators embedded in tires and wheels for monitoring air pressure and tire conditions. In this project we want to analyze the effects of electromagnetic radiation on living system. We want to perform the experiment on Mimosa pudica seeds. The reason for selecting Mimosa pudica seeds for the experiment is that they assure the fast growth. The objective of the project is to carry the effects of electromagnetic radiation based on various parameters. The methods followed by analyzing the radiation effects are discussed.

\subsection{Radiating The Seeds:}

\section{Methodology}

The seeds which are collected are using a microwave bench setup and horn antenna. The setup works in microwave $\mathrm{x}$-band range and the frequency of the microwaves is found to be $10 \mathrm{MHz}$.

The seeds are divided into six categories:

1. Seeds that are not radiated.

2. Seeds that are radiated for $10 \mathrm{~min}$.

3. Seeds that are radiated for $20 \mathrm{~min}$

4. Seeds that are radiated for $30 \mathrm{~min}$

5. Seeds that are radiated for $45 \mathrm{~min}$ 
6. Seeds that are radiated for $60 \mathrm{~min}$

\subsection{Planting The Seeds:}

Plants grown in pots mixture of sandy, gravel and peat, were used. Plants were grown in a greenhouse at $15-22^{\circ} \mathrm{c}$, in normal humidity and without artificial light.

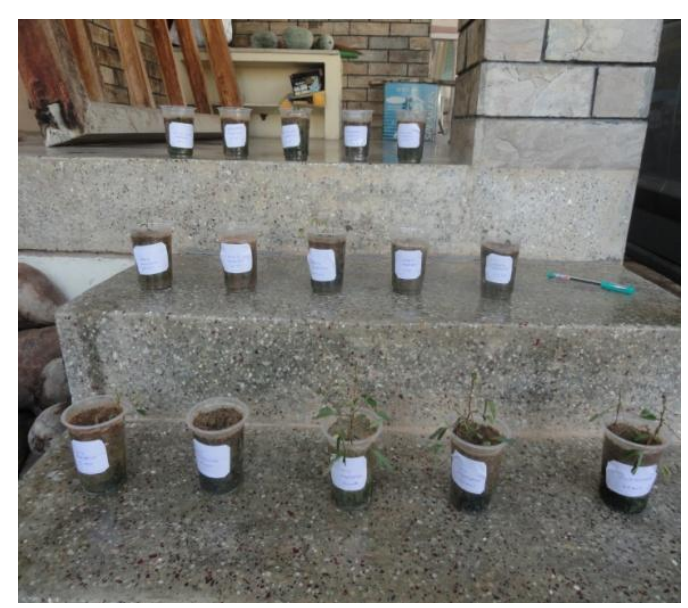

Fig: 1 Mimosa pudica plants

\subsection{Analysis Of Electromagnetic Radiation Effects On Plants Based On Physical Growth :}

In this type of analysis we want to analyze the effects of electromagnetic radiations based on physical growth. The growth of the plant is analyzed based on the size of the leaf, leaflet size and closing of leaves. Averaging concept is used for more accurate results.

\subsection{Comparision Of Closing Of Leaves:}

The leaf is attached to its stalk exhibited the opening-closing movements in response to light stimulis. Closing leaves in response to physical stimuli present several energetic challenges for mimosa pudica. The leaf folding behavior of mimosa pudica has benefits for the plant. The leaf folding was a mechanism for reducing nutrient loss in response to rainfall.

\subsection{Comparision Of Leaf Let Size:}

The leaf let are measured for every week. In order to achieve better accuracy have used averaging concept. We have measured the leaflet of five leaves one from each plant of every sample and averaged them, which gives average leaflet of leaf. The access of the leaf movement quantitatively, we devised. This tool can be folded small in order to adjust to the opening angle between the main vein of a leaflet and stalk of the excised leaf, and makes the measurement of the leaflet opening angle and quick and accurate.

\section{Circuit Design For Action Potential}

The values of action potentials of living organisms are very small. Hence we are using a transducer unit for visualizing the signal in a better way. The design of transducer unit is discussed in this chapter. The design of development transducer unit includes three subunits:

1.A high sense amplifier

2. A Butterworth filter

3. A voltage to current converter

\subsection{High Sense Amplifier:}

An amplifier is a device for increasing the power of a signal by use of an external energy source. Here we are using voltage amplifier. Generally the action potentials of the plants are very small. Hence we need a high sense amplifier for amplifying this voltage. The simulation of the high sense amplifier is carried using top spice simulation software. The schematic drawn in the top spice simulation is as shown. 


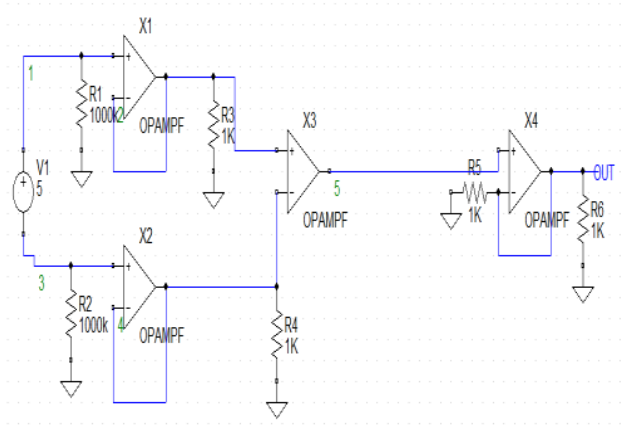

Fig 2: high sense amplifier -schematic diagram

The high sense amplifier is very much essential for the amplification of the small voltages. The graph of the amplifier output versus amplifier input is as shown.

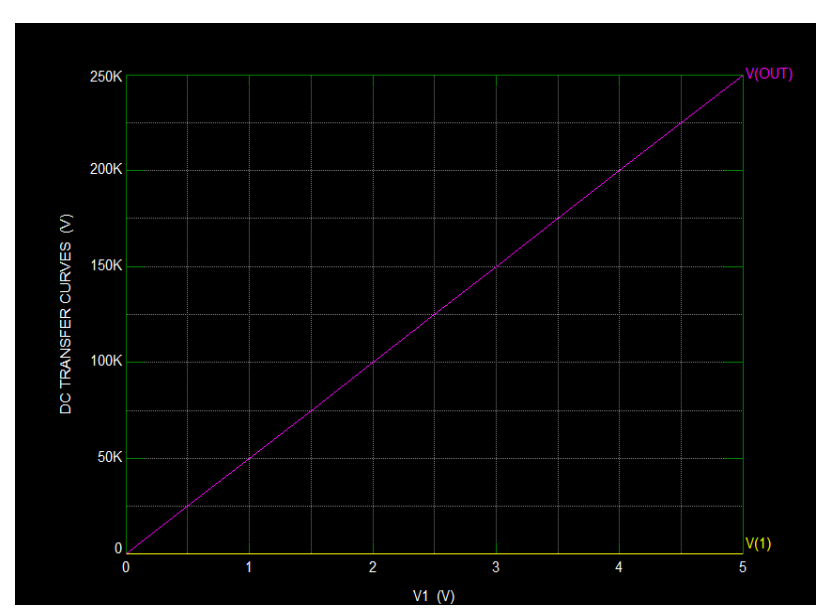

Fig 3 : High Sense Amplifier- Input Signal Vs Output Signal

\section{2 $8^{\mathrm{TH}}$ ORDER BUTTERWORTH FILTER:}

Amplifier input is given to butterworth filter. The butterworth filter is very essential in transducer unit. Due to high amplification the noise levels also may raise. To filter the unwanted noise components we need a butterworth filter. For good filtering we are using $8^{\text {th }}$ order Butterworth filter.

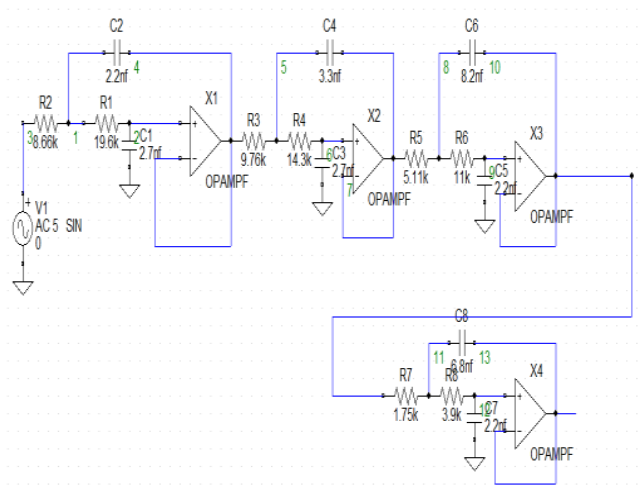

Fig $4: 8^{\text {th }}$ order buttrworth filter- schematic diagram

The butter worth filter designed with a cutoff frequency $2 \mathrm{KHz}$.The frequency response of the Butterworth filter obtained from the simulation as shown. 


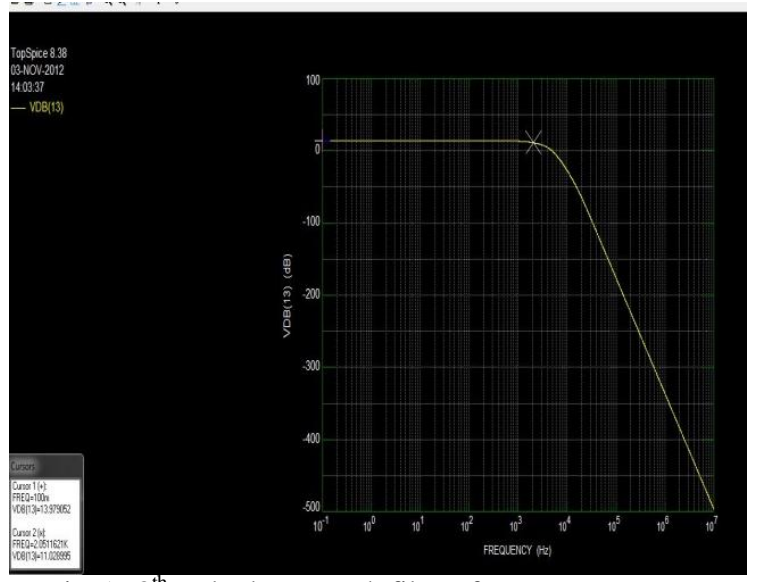

Fig $5: 8^{\text {th }}$ order buttrworth filter- frequency response

\subsection{Voltage To Current Converter:}

A voltage to current converter is also used in the transducer. . For transmission, was designed a high sense Voltage to Current converter. The circuit is shown in Fig. 7 is used so that the signal could be transmitted reliably.

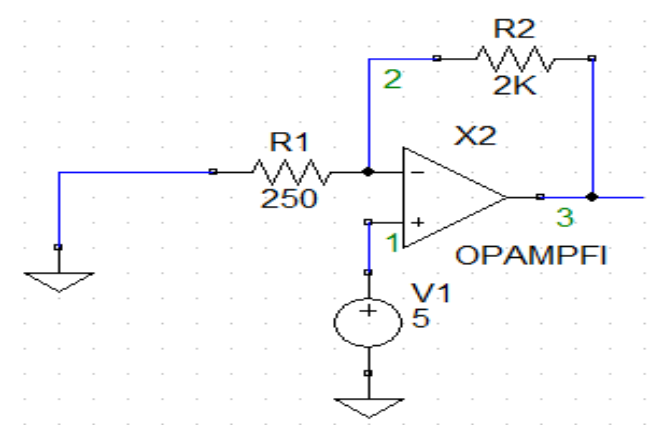

Fig 6:Voltage to current converter-schematic diagram

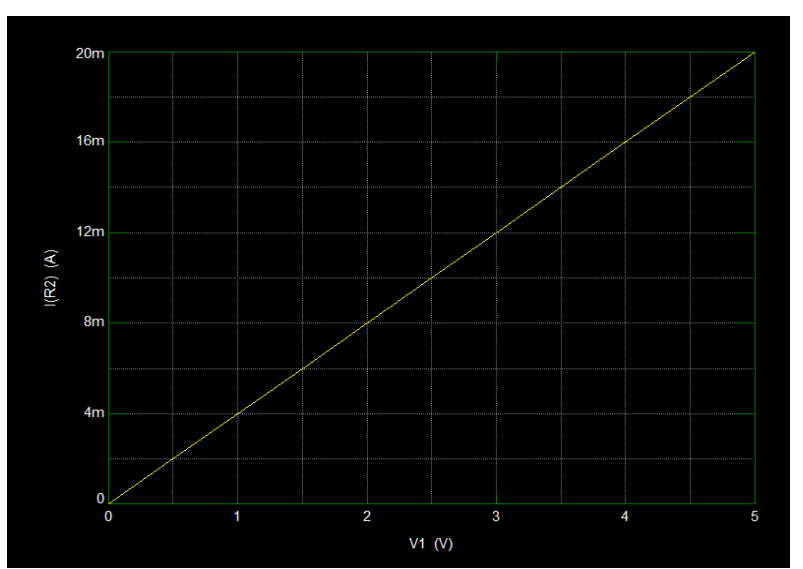

Fig 7: Voltage to current converter-input voltage vs output current

\section{Conclusion}

The communication plays a major role in our day to day life. Microwaves play a key role in various communication technologies now days. But these microwaves are harmful to living organisms. From the above analysis we know that microwave radiation shows a negative impact on plants growth. From the image histograms we can observe that the intensity of green component is more in no radiated leaf than the others. The green component intensity of leaf decreases as time of radiation increases. We can conclude based on image thresholding results that the spots are increasing as the radiation time is more. The analysis based on action potentials will be done and the results will be compared with biological analysis. 


\section{REFERENCES}

\section{Journal Papers:}

[1]. Jensen, Evelyn L., Lawrence M. Dill, and James F. Cahill Jr. "Applying behavioral-ecological theory to plant defense: Lightdependent movement in Mimosa pudica suggests a trade-off between predation risk and energetic reward." The American Naturalist 177.3 (2011): 377-381.

[2]. Jackson, Douglas J., et al. "Portable high-voltage power supply and electrochemical detection circuits for microchip capillary electrophoresis."Analytical chemistry 75.14 (2003): 3643-3649.

[3]. Neshatvar, Nazanin. DESIGNING LOW FREQUENCY IC FILTER USING PSEUDO RESISTOR MEASURMENTS. Diss. American University, 2010.

FOR BIOPOTENTIAL

[4]. Kocher, Mark J., and Robert L. Steigerwald. "An AC-to-DC converter with high quality input waveforms." Industry Applications, IEEE Transactions on 4 (1983): 586-599. 